\title{
Race/ethnicity and other risk of factors associated with cryptosporidiosis as an initial AIDS-defining condition in California, 1980-99
}

\author{
A. KHALAKDINA ${ }^{1,2}$, F. TABNAK ${ }^{3}$, R. K. P. SUN ${ }^{3}$ AND J. M. COLFORD, JR. ${ }^{1 *}$ \\ ${ }^{1}$ University of California, Berkeley, School of Public Health, Division of Public Health Biology and \\ Epidemiology, Center for Family and Community Health, Center for Occupational and Environmental Health, \\ Berkeley, CA 94720-7360, USA \\ ${ }^{2}$ Community Health Epidemiology and Disease Control, San Francisco Department of Public Health, 25 Van \\ Ness Ave. \#710, San Francisco, CA 94102-6033, USA \\ ${ }^{3}$ HIV / AIDS Epidemiology Branch, Office of AIDS, California Department of Health Services, 611 North 7th \\ St., P.O. Box 942732, Sacramento, CA 94234-7320, USA
}

(Accepted 12 July 2001)

\section{SUMMARY}

To study whether African-Americans are less likely than whites to present with cryptosporidiosis as an AIDS-defining condition (ADC), a case-control study was conducted using a large, population-based surveillance registry of AIDS patients in California. Data from January 1980 through June 1999 were analysed using risk factor stratification and multivariate logistic regression to evaluate confounding by other risk factors such as gender, injection drug use (IDU), CD4 counts, age and sexual orientation. Cases included 1373 subjects with cryptosporidiosis as an ADC and controls included 97419 subjects with other ADC. The results indicate a significantly lower risk for presentation with cryptosporidiosis as an ADC among African-Americans compared with whites (OR vs. whites $=0.5,95 \%$ CI 0.4, 0.7). Additionally, there is evidence that heterosexuals are less likely than homosexual/bisexual males to present with cryptosporidiosis $(\mathrm{OR}=0 \cdot 5,95 \% \mathrm{CI} 0 \cdot 4,0 \cdot 7)$. Our analyses also suggest a decreasing risk with increasing age. The possibility that there may be biologic factors or differential lifetime exposures that account for the difference between the racial/ethnic groups merits further investigation.

\section{INTRODUCTION}

Cryptosporidiosis is a gastrointestinal infectious disease that can be life threatening in immunocompromised individuals [1]. There are no effective therapies that eliminate the parasite although some have been found to alleviate disease severity [2]. It is estimated that $10-15 \%$ of patients in the United

\footnotetext{
* Author for correspondence: Division of Public Health Biology and Epidemiology, School of Public Health, 140 Warren Hall \# 7360, University of California, Berkeley, Berkeley, CA 947207360 , USA.
}

States with acquired immunodeficiency syndrome (AIDS) will develop chronic infection with Cryptosporidium [3]. The organism is found in the environment, especially in water bodies and among livestock, and is a common cause of human diarrhoea worldwide.

One mode of transmission of this pathogen is via water, particularly during epidemics, but other routes such as person-to-person spread account for a considerable portion of low-level, endemic cryptosporidiosis in the community [4]. Earlier studies among immunocompromised individuals have 
suggested that African Americans are at reduced risk for acquiring cryptosporidiosis [5-8], as are heterosexuals (compared to men-who-have-sex-with-men (MSM)) [6, 9]. A recent report for AIDS opportunistic infections highlighted the fact that Cryptosporidium infects significantly more MSM as an initial diagnosis and during the course of AIDS than injection drug users (IDUs) and heterosexuals [10]. Because the sample sizes of prior studies were small it is not known whether the observed racial difference may be attributable to confounding factors such as differences in the distribution of sexual orientation between the racial/ethnic groups or between other risk factors for acquiring cryptosporidiosis.

The purpose of this investigation was to identify the risk factors for presenting with cryptosporidiosis as an AIDS-defining condition. Additionally, our aim was to determine whether there were any differences among the major demographic groups in California, particularly between racial/ethnic groups, and among those with different modes of exposure to HIV infection. The California population-based AIDS surveillance registry provided the sample size necessary to address these research questions. Specifically, we evaluated whether or not confounding by other risk factors such as gender, injection drug use (IDU), CD4 counts, age and sexual orientation was present.

\section{MATERIALS AND METHODS}

\section{Sampling frame}

The HIV/AIDS Reporting System (HARS) registry is a national surveillance system for AIDS cases maintained by the Centers for Disease Control and Prevention (CDC). The California Department of Health Services (CDHS) maintains a registry with demographic and clinical data on incident and prevalent AIDS as reported by local health departments. For each patient, multiple variables are recorded, including date of birth, date of AIDS diagnosis, gender, race/ethnicity, mode of exposure to HIV, specific AIDS-defining condition(s) with date(s) of diagnosis, date of death (if deceased) and CD4 lymphocyte counts. The data analysed in this study were extracted from the California HARS (1 January, 1980 through 1 July 1999); a total of 112931 cases of AIDS were reported during this period.

This research study was granted human subjects approval by the institutional review board for University of California, Berkeley. Informed consent was not obtained from individual subjects since these data did not include any personal identifying information for any of the subjects.

\section{Selection of cases and controls}

Even though the California HARS is an active surveillance system that collects information on incident AIDS cases, there is wide variability at the local health department level in the collection of follow-up data on these cases, such as the development of subsequent opportunistic infections or current CD4 counts. Therefore, to avoid potential reporting bias differences, we compared presenting cases of cryptosporidiosis ('cases') to AIDS cases that were never reported to have had a diagnosis of cryptosporidiosis at any time ('controls'). A 'presenting' case of cryptosporidiosis is a case that had an AIDS-defining condition of cryptosporidiosis as their initial diagnosis. To examine whether there were any fundamental differences between presenting and nonpresenting cryptosporidiosis AIDS cases the baseline characteristics of the two were compared.

\section{Inclusion criteria}

Cases were selected for inclusion if cryptosporidiosis was their initial AIDS-defining condition (ADC). All cases that had a cryptosporidiosis diagnosis date after their AIDS diagnosis date were discarded as they represented secondary cryptosporidiosis cases. Controls $(n=97419)$ were all those AIDS cases remaining after eliminating presenting $(n=1373)$ and non-presenting cryptosporidiosis cases.

\section{Exclusion criteria}

The variables used in the analysis were diagnosis dates for AIDS and cryptosporidiosis, gender, race/ ethnicity and mode of HIV exposure. We retained only those who were of white $(n=64722)$, African American $(n=15835)$, or Hispanic $(n=18235)$ race/ ethnicity; and were adult/adolescent cases (over 12 years of age at diagnosis; paediatric cases $n=578$ ). The other racial/ethnicity categories (Asian Pacific Islander and Native Americans) contained numbers too small for meaningful analysis $(n=2964)$. Of the 3586 cases of cryptosporidiosis reported in HARS as of 1 July 1999, $1373(38.3 \%)$ were presenting cases with complete information. Of the 109345 noncryptosporidiosis cases in the data set, 97419 (89.1\%) 
were retained in the analysis as controls with complete information.

\section{Analyses \\ Univariate}

Odds ratios were used as estimates of relative risks for presentation with cryptosporidiosis as an AIDSdefining condition. Univariate ORs were calculated for each variable using Stata 6.0 (Stata Corporation, College Station, TX) with $95 \%$ CI. These variables were: gender (male/female); sexual orientation (MSM or not MSM); injection drug use (yes/no); age group (mid-decade intervals), CD4 counts closest to AIDS diagnosis (dichotomized at median for cases), and race/ethnicity (white, African American, Hispanic). Test for trend in age group was performed using the Cochran-Armitage trend test in SAS 6.12 (SAS Institute Inc., Cary, NC). Non-parametric WilcoxonMann-Whitney tests were employed to test for differences in median age and CD4 counts between the cases and controls.

\section{Risk factor stratification}

Variables significant in the univariate analysis (except for age-group) were analysed by comparing all possible combinations (i.e. covariate patterns) of gender, IDU, sexual orientation and race/ethnicity. The reference group for all the others was homosexual/bisexual, male, white, non-IDU; according to previous reports this group should be at highest risk for cryptosporidiosis [5-9]. Stratification by age group was not done because of the large number of categories that would have produced data too sparse for meaningful analysis.

\section{Multivariate logistic regression}

To adjust for confounding, a multivariate main effects (additive) logistic regression model using SAS 6.12 was constructed with the covariates that were significant in the univariate analysis. These included gender, race/ethnicity, MSM, IDU, and age group. The Hosmer-Lemeshow test was conducted to assess goodness-of-fit of the fitted model values to the observed data [11].

A further set of analyses (including univariate ORs, risk factor stratification and multivariate logistic regression) was conducted using the subset of cases and controls for whom CD4 counts and dates were available (615 cases and 58007 controls). The CD4 count with date closest to the AIDS-diagnosis date was used. CD4 count was dichotomized ('higher' versus 'lower') at the median value for cryptosporidiosis cases so as to impose no assumptions about the distribution.

\section{RESULTS}

In California, 3.3\% (3586/109345) of all AIDS patients ever reported to the HARS registry were infected with Cryptosporidium from January 1980 through June 1999 (Fig. 1). The greatest number of cryptosporidiosis cases $(n=211)$ were reported in 1991, and cryptosporidiosis as a percentage of all AIDS peaked at $4.4 \%$ in the 4th quarter of 1983. During the study period, a total of 1373 cases with cryptosporidiosis as an AIDS-defining condition and 97419 AIDS cases without a primary or subsequent diagnosis of cryptosporidiosis, i.e. controls, met the inclusion criteria. Of these, $615(44 \cdot 8 \%)$ cases and $58007(59 \cdot 5 \%)$ controls had CD4 counts and dates of CD4 counts available for further analysis.

\section{Baseline characteristics}

\section{Univariate}

Table 1 presents the baseline characteristics for the cryptosporidiosis and non-cryptosporidiosis AIDS cases during the study period. Significantly fewer women than men presented with cryptosporidiosis as their AIDS-defining condition (OR $=0.4,95 \% \mathrm{CI}$ $0 \cdot 3,0 \cdot 6)$. In comparison to whites, African Americans had significantly lower risk for presenting with cryptosporidiosis $\quad(\mathrm{OR}=0.5,95 \%$ CI $0.4,0.6)$. Hispanics had a risk similar to whites $(\mathrm{OR}=0 \cdot 9$, $95 \%$ CI $0 \cdot 8,1 \cdot 1)$. With regard to age, there appeared to be a trend of decreasing risk with increasing age (Cochran-Armitage trend test $P$-value $=0 \cdot 001$ ). The median age for those with cryptosporidiosis (35 years, interquartile range (IQR) 31-41 years) was similar to that for those without (37 years, IQR 32-44 years). Although this difference was statistically significant due to large sample sizes (Wilcoxon-Mann-Whitney test, $P$-value $<0.0001)$, it is not clinically relevant. Individuals who were less than 35 years of age were significantly more likely to present with cryptosporidiosis as their AIDS-defining diagnosis $(\mathrm{OR}=$ $1 \cdot 4,95 \%$ CI $1 \cdot 3,1 \cdot 6$ (data not shown)).

We analysed two of the known risk groups for HIV infection: IDUs and MSM. In the univariate analysis, 


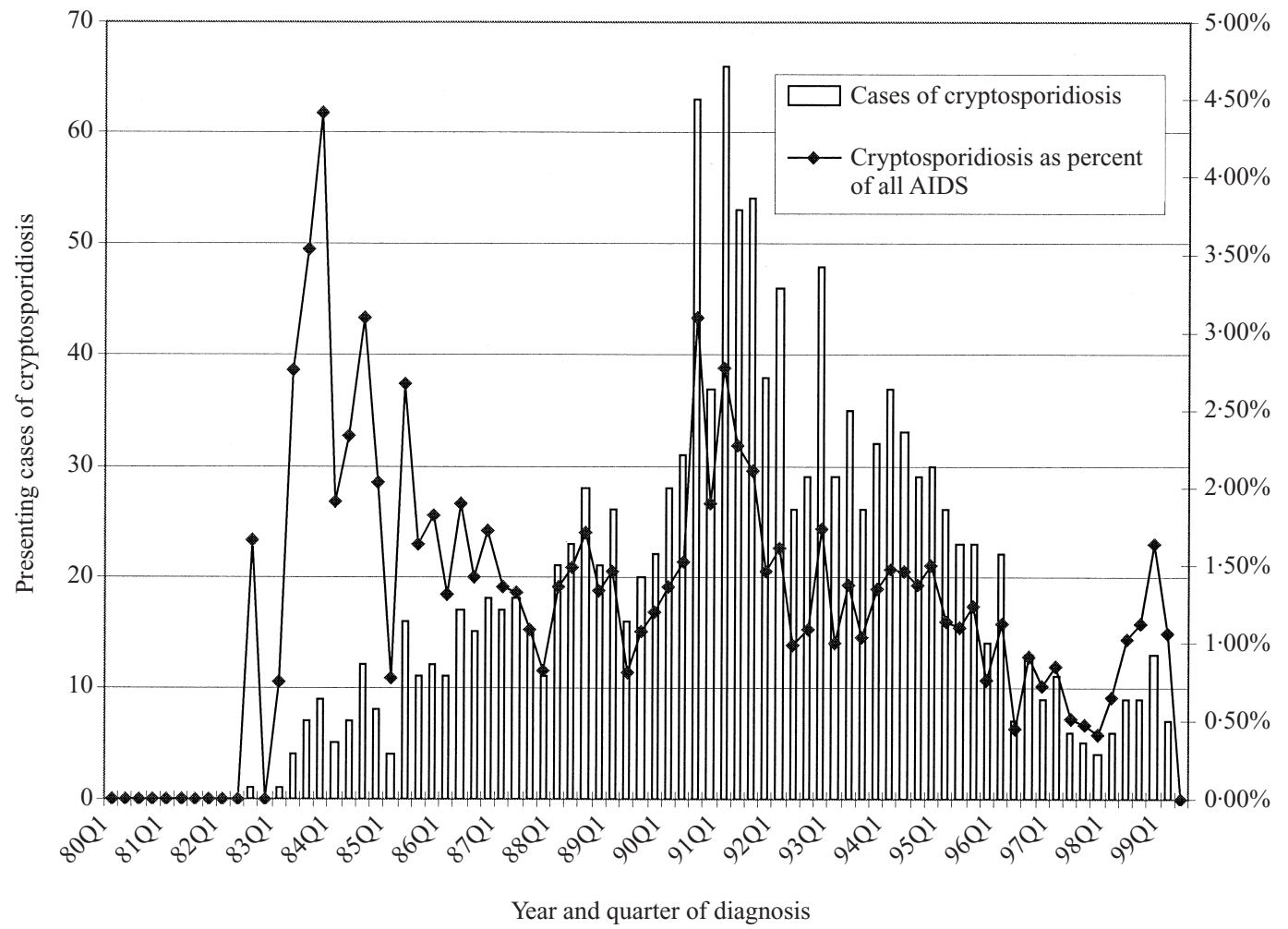

Fig. 1. Temporal trend of cryptosporidiosis as an AIDS-defining condition in California, 1980-99. Number of presenting cases of cryptosporidiosis overall (units on left axis and represented by bars) and cryptosporidiosis as a percentage of all other AIDS-defining conditions (units on right axis and represented by line) by year and quarter (YYQ) of AIDS diagnosis for the cases.

those who had IDU exposure were at a significantly lower risk for presenting with cryptosporidiosis as their AIDS-defining condition than those who did not have IDU exposure (OR $=0.7,95 \%$ CI $0.6,0.8)$; similarly, cases who had heterosexual contact were at a significantly lower risk $(\mathrm{OR}=0 \cdot 4,95 \%$ CI $0 \cdot 3,0 \cdot 5)$.

Additional analysis of the subset of AIDS cases for whom CD4 counts were available produced similar results to those for the overall study population (data not shown): there were significantly fewer females $(\mathrm{OR}=0 \cdot 4,95 \% \mathrm{CI} 0 \cdot 2,0 \cdot 6)$; African Americans (OR $=0.4,95 \%$ CI $0.3,0 \cdot 6)$; IDUs $(\mathrm{OR}=0.8,95 \% \mathrm{CI}$ $0 \cdot 6,0.9)$; and heterosexuals ( $\mathrm{OR}=0.4,95 \%$ CI $0 \cdot 3$, 0.5). The median CD4 count was 109 cells $/ \mathrm{ml}$ (IQR $38-270$ cells $/ \mathrm{ml}$ ) and 130 cells $/ \mathrm{ml}$ (IQR 50190 cells $/ \mathrm{ml}$ ) for cases and controls, respectively. However, the distribution of CD4 counts was not statistically different for cases and for controls (Wilcoxon-Mann-Whitney, $P$-value $<0.44$ ) probably because of a highly skewed distributions (means, compared to the medians, were mean $=183 \mathrm{vs}$. median $=109$ for cases, and mean $=143 \mathrm{vs}$. median $=130$ for controls). Comparison of cases with relatively lower CD4 counts dichotomized at the median for cases $(109$ cell $/ \mathrm{ml})$ versus those with higher CD4 counts revealed that the former were significantly more likely to present with cryptosporidiosis as an AIDS-defining condition (OR $=1 \cdot 3,95 \% \mathrm{CI} 1 \cdot 1,1 \cdot 5)$.

We also analysed AIDS cases that had cryptosporidiosis as a secondary or subsequent diagnosis (data not shown); there were no differences in the inferences drawn from these results. Additionally, to assess whether there were any temporal influences on the risk estimates, we conducted univariate analyses for the major variables by separating into those before and those after the median date of diagnosis for presenting cryptosporidiosis AIDS cases (July 1991). The ORs were very similar for each time period and for the combined data (data not shown), suggesting that date of diagnosis has not biased our results.

\section{Risk factor stratification}

Table 2 presents the ORs for all possible covariate patterns for all cases and controls. In this table white, male, homosexual/bisexual, non-IDU is the baseline comparison group for all the other patterns. Among whites, all females (IDU: OR $=0 \cdot 3,95 \%$ CI $0 \cdot 1,0 \cdot 7$; 
Table 1. Baseline characteristics, and univariate and multivariate logistic regression analyses of case and control AIDS patients from the California HARS registry, 1980-99

\begin{tabular}{|c|c|c|c|c|c|c|}
\hline & \multicolumn{2}{|c|}{$\begin{array}{l}\text { Presenting Cryptosporidium } \\
\text { infected cases }\end{array}$} & \multicolumn{2}{|c|}{$\begin{array}{l}\text { Non-Cryptosporidium } \\
\text { infected cases }\end{array}$} & \multirow{2}{*}{$\begin{array}{l}\text { Univariate OR } \\
(95 \% \mathrm{CI})\end{array}$} & \multirow{2}{*}{$\begin{array}{l}\text { Multivariate OR } \\
(95 \% \mathrm{CI})\end{array}$} \\
\hline & No. & $\%$ & No. & $\%$ & & \\
\hline \multicolumn{7}{|l|}{ Gender } \\
\hline Male & 1344 & $97 \cdot 9$ & 92327 & $94 \cdot 8$ & $1 \cdot 0$ & $1 \cdot 0$ \\
\hline Female & 29 & $2 \cdot 1$ & 5092 & $5 \cdot 2$ & $0 \cdot 4(0 \cdot 3,0 \cdot 6)^{*}$ & $0 \cdot 8(0 \cdot 52,1 \cdot 3)$ \\
\hline \multicolumn{7}{|l|}{ Race/ethnicity $\dagger$} \\
\hline White & 995 & $65 \cdot 4$ & 63727 & $72 \cdot 5$ & $1 \cdot 0$ & $1 \cdot 0$ \\
\hline African American & 118 & $16 \cdot 1$ & 15717 & $8 \cdot 6$ & $0.5(0 \cdot 4,0.6)^{*}$ & $0 \cdot 5(0 \cdot 4,0 \cdot 6)$ \\
\hline Hispanic & 260 & $18 \cdot 5$ & 17975 & $18 \cdot 9$ & $0 \cdot 9(0 \cdot 8,1 \cdot 1)$ & $0 \cdot 9(0 \cdot 8,1 \cdot 0)$ \\
\hline \multicolumn{7}{|l|}{ Age (years)ł§ } \\
\hline $13-24$ & 51 & $3 \cdot 7$ & 2431 & $2 \cdot 5$ & $1 \cdot 5(1 \cdot 1,2 \cdot 0)^{*}$ & $1 \cdot 7(1 \cdot 2,2 \cdot 2)^{*}$ \\
\hline $25-34$ & 562 & $40 \cdot 9$ & 33212 & $34 \cdot 1$ & $1 \cdot 2(1 \cdot 1,1 \cdot 3)^{*}$ & $1 \cdot 2(1 \cdot 1,1 \cdot 4)^{*}$ \\
\hline $35-44$ & 559 & $40 \cdot 7$ & 39392 & $40 \cdot 4$ & $1 \cdot 0$ & $1 \cdot 0$ \\
\hline $45-54$ & 165 & $12 \cdot 0$ & 16196 & $16 \cdot 6$ & $0.7(0.6,0.9)^{*}$ & $0.7(0.6,0.8)^{*}$ \\
\hline $55-64$ & 27 & $2 \cdot 0$ & 4866 & $5 \cdot 0$ & $0 \cdot 4(0.3,0 \cdot 6)^{*}$ & $0.4(0.3,0.6)^{*}$ \\
\hline$>65$ & 9 & $0 \cdot 7$ & 1322 & $1 \cdot 4$ & $0 \cdot 5(0 \cdot 3,0 \cdot 9)^{*}$ & $0 \cdot 5(0 \cdot 3,1 \cdot 0)$ \\
\hline \multicolumn{7}{|c|}{ CD4 counts (cells/ml)\| } \\
\hline 109 or more & 308 & $50 \cdot 1$ & 32802 & $56 \cdot 6$ & $1 \cdot 0$ & $1 \cdot 0$ \\
\hline less than 109 & 307 & $49 \cdot 9$ & 25205 & $43 \cdot 4$ & $1 \cdot 3(1 \cdot 1,1 \cdot 5)^{*}$ & $1 \cdot 3(1 \cdot 2,1 \cdot 6)$ \\
\hline \multicolumn{7}{|l|}{ Injection drug use } \\
\hline No & 1191 & $86 \cdot 7$ & 79956 & $82 \cdot 1$ & $1 \cdot 00$ & $1 \cdot 0$ \\
\hline Yes & 182 & $13 \cdot 3$ & 17463 & $17 \cdot 9$ & $0 \cdot 70(0 \cdot 60,0 \cdot 82)^{*}$ & $0 \cdot 9(0 \cdot 8,1 \cdot 0)$ \\
\hline \multicolumn{7}{|l|}{ Sexual orientation } \\
\hline MSM\# & 1293 & $94 \cdot 2$ & 84490 & $86 \cdot 7$ & $1 \cdot 00$ & $1 \cdot 0$ \\
\hline Heterosexual & 80 & $5 \cdot 8$ & 12929 & $13 \cdot 3$ & $0 \cdot 40(0 \cdot 32,0 \cdot 51)^{*}$ & $0.5(0.4,0.7)^{*}$ \\
\hline Total & 1373 & $1 \cdot 4$ & 97419 & $98 \cdot 6$ & & \\
\hline
\end{tabular}

* $P$-value (probability of this result or a more extreme one occurring by chance alone) $<0.05$.

$\dagger$ This study was limited to only African Americans, whites, and Hispanics.

$\$$ Pediatric cases (12 years and under) were removed from the analysis.

$\S$ Cochran-Armitage trend test $P$-value $=0.001$.

- Median CD4 count for cases $=109$ cells $/ \mathrm{ml}$.

\| Data presented are for the subset of cases with CD4 counts available (615 cases and 58007 controls).

\# Men who have sex with men.

non-IDU: $\mathrm{OR}=0 \cdot 3,95 \% \mathrm{CI} 0 \cdot 1,0 \cdot 6)$ and males who were heterosexual, IDUs (OR $=0 \cdot 4,95 \%$ CI $0 \cdot 2,0 \cdot 6)$ were at a significantly lower risk for presenting with cryptosporidiosis. Each covariate pattern among African Americans revealed that the risk of presentation with cryptosporidiosis was significantly lower for these individuals when compared with the baseline group; this trend was the same for each pattern when compared to its white counterpart. Overall, African American females were at lowest risk for cryptosporidiosis (non-IDU: OR $=0 \cdot 1,95 \%$ CI $0 \cdot 0,0 \cdot 5$; IDU: $\mathrm{OR}=0 \cdot 2,95 \% \mathrm{CI} 0 \cdot 1,0 \cdot 5)$, after heterosexual, non-IDU males $(\mathrm{OR}=0 \cdot 1,95 \% \mathrm{CI} 0 \cdot 0,0 \cdot 8)$. African American, IDU, heterosexual males had a risk that was one-fifth that of the baseline group ( $\mathrm{OR}=0 \cdot 2$, $95 \%$ CI 0·1, 0.4). Fewer homosexual/bisexual African American males had an AIDS-defining condition of cryptosporidiosis whether IDU $(\mathrm{OR}=0 \cdot 6,95 \% \mathrm{CI}$ $0.4,1.0)$ or not $(\mathrm{OR}=0.6,95 \%$ CI $0.5,0.7)$. The differences between the white, male, non-IDU, homosexual/bisexual group and the Hispanic strata were not significant for any group.

For each category of covariate patterns we compared African Americans and Hispanics to whites (data not shown). Only three of the African American strata were at significantly lower risk of presenting with cryptosporidiosis than their White counterparts: MSM who were IDU $(\mathrm{OR}=0 \cdot 6,95 \% \mathrm{CI} 0 \cdot 4,1 \cdot 0)$ and 
Table 2. Stratified risk factor analysis: odds ratios and frequencies for all AIDS cases with cryptosporidiosis (crypto) as an AIDS-defining condition and non-cryptosporidiosis (non-crypto) AIDS cases with other diagnoses from the California HARS registry, 1980-1999†

\begin{tabular}{|c|c|c|c|c|c|c|c|c|c|}
\hline & \multicolumn{3}{|c|}{ White $(n=64722)$} & \multicolumn{3}{|c|}{ African American $(n=15835)$} & \multicolumn{3}{|c|}{ Hispanic $(n=18235)$} \\
\hline & Crypto & $\begin{array}{l}\text { Non- } \\
\text { Crypto }\end{array}$ & $\begin{array}{l}\text { OR } \\
(95 \% \mathrm{CI})\end{array}$ & Crypto & $\begin{array}{l}\text { Non- } \\
\text { Crypto }\end{array}$ & $\begin{array}{l}\text { OR } \\
(95 \% \mathrm{CI})\end{array}$ & Crypto & $\begin{array}{l}\text { Non- } \\
\text { Crypto }\end{array}$ & $\begin{array}{l}\text { OR } \\
(95 \% \mathrm{CI})\end{array}$ \\
\hline \multicolumn{10}{|l|}{ Male } \\
\hline \multicolumn{10}{|l|}{ MSM } \\
\hline Non-IDU§ & 855 & 52160 & $1 \cdot 0 \dagger$ & 85 & 9152 & $0.6(0 \cdot 5,0 \cdot 7)^{*}$ & 213 & 13724 & $1 \cdot 0(0 \cdot 8,1 \cdot 1)$ \\
\hline IDU & 104 & 6165 & $1 \cdot 0(0 \cdot 8,1 \cdot 3)$ & 19 & 1828 & $0.6(0.4,1 \cdot 0)^{*}$ & 17 & 1461 & $0 \cdot 7(0 \cdot 4,1 \cdot 1)$ \\
\hline \multicolumn{10}{|l|}{ Heterosexual } \\
\hline Non-IDU & 14 & 1085 & $0 \cdot 8(0 \cdot 5,1 \cdot 3)$ & 1 & 461 & $0 \cdot 1(0 \cdot 0,0 \cdot 8)^{*}$ & 3 & 497 & $0 \cdot 4(0 \cdot 1,1 \cdot 1)$ \\
\hline IDU & 13 & 2228 & $0.4(0 \cdot 2,0 \cdot 6)^{*}$ & 8 & 2345 & $0 \cdot 2(0 \cdot 1,0 \cdot 4)^{*}$ & 12 & 1221 & $0 \cdot 6(0 \cdot 3,1 \cdot 1)$ \\
\hline \multicolumn{10}{|l|}{ Female } \\
\hline \multicolumn{10}{|l|}{ Heterosexual } \\
\hline Non-IDU & 5 & 1223 & $0 \cdot 3(0 \cdot 1,0 \cdot 6)^{*}$ & 2 & 880 & $0 \cdot 1(0 \cdot 0,0 \cdot 5)^{*}$ & 13 & 774 & $1 \cdot 0(0 \cdot 6,1 \cdot 8)$ \\
\hline IDU & 4 & 866 & $0 \cdot 3(0 \cdot 1,0 \cdot 7)^{*}$ & 3 & 1051 & $0 \cdot 2(0 \cdot 1,0 \cdot 5)^{*}$ & 2 & 298 & $0 \cdot 4(0 \cdot 0,1 \cdot 5)$ \\
\hline Total & 995 & 63727 & & 118 & 15717 & & 260 & 17975 & \\
\hline
\end{tabular}

* $P$-value $<0 \cdot 05$.

$\dagger$ White, Male, MSM, Non-IDU is the reference category for all strata in the table.

$\$$ Men who have sex with men.

$\S$ Injection Drug User.

non-IDU $(\mathrm{OR}=0.6,95 \%$ CI $0 \cdot 5,0 \cdot 7)$, and heterosexual, non-IDU, males $(\mathrm{OR}=0 \cdot 2,95 \%$ CI $0 \cdot 0$, $1 \cdot 0)$. Hispanic, non-IDU females were at a significantly higher risk than their white counterparts (OR $=4 \cdot 1,95 \%$ CI $1 \cdot 5,11 \cdot 1)$.

The analysis of the subset of those AIDS cases with CD4 counts available used white, male, homosexual/ bisexual, non-IDU with lower CD4 counts as the comparison group for all the others (data not shown). Both African American and white injection drug using, heterosexual males are at a significantly lower risk regardless of higher (white $\mathrm{OR}=0 \cdot 2,95 \%$ CI $0 \cdot 1$, 0.7; African American OR $=0 \cdot 2,95 \%$ CI 0.0, 0.6) or lower (white OR $=0.3,95 \%$ CI $0.1,0.9$; African American OR $=0 \cdot 4,95 \%$ CI $0 \cdot 2,1 \cdot 0)$ CD4 counts. Another group where consistently fewer cases presented with cryptosporidiosis was African American, MSM, non-IDU males with higher (OR = $0 \cdot 4,95 \%$ CI $0 \cdot 2,0 \cdot 6)$ and lower (OR $=0 \cdot 6,95 \% \mathrm{CI}$ $0.4,0.9) \mathrm{CD} 4$ counts. In each of the categories mentioned those with higher CD4 counts were less likely to have an AIDS diagnosis of cryptosporidiosis than their counterparts with lower CD4 counts. Except for MSM, non-IDU males with higher CD4 counts $(\mathrm{OR}=0.5,95 \%$ CI $0.4,0.7)$ none of the Hispanic covariate patterns displayed a significantly different risk from the baseline group.

\section{Multivariate logistic regression}

Table 1 also shows the results of the multivariate logistic regression model that was constructed using the variables gender, race/ethnicity, IDU, sexual orientation and age group. Odds ratios adjusted for all other variables in the model indicated that African Americans were at a significantly lower risk $(\mathrm{OR}=$ $0 \cdot 5,95 \% \mathrm{CI} 0 \cdot 4,0 \cdot 6)$ as were heterosexuals ( $\mathrm{OR}=0 \cdot 5$, $95 \%$ CI $0.4,0.7)$. Similar to the univariate analysis, age groups younger than 35 years of age were at significantly higher risk and those older than 44 years of age were at lower risk compared to the baseline group of 35-44 year olds. The model for the subset with CD4 counts produced almost identical ORs as that for the entire population.

\section{Goodness-of-fit for the logistic model}

Odds ratios for each covariate pattern were derived from the multivariate logistic model and compared to the observed ORs (data not shown). The differences were slight and did not alter the inferences drawn. More formally, we examined the fit of the models using Hosmer-Lemeshow goodness-of-fit tests. For the overall population and for those with CD4 count the $P$-values were 0.196 and 0.711 , respectively (null hypothesis: model provides a good fit to the data). 


\section{DISCUSSION}

This investigation confirms earlier reports from Los Angeles and San Francisco that African Americans are less likely than whites to present with cryptosporidiosis as an AIDS-defining condition [6-8]. A Texas study reported that Hispanics were at a higher risk than whites, which they conjectured could be a result of frequent travel and exposure in childhood [12]. In this study Hispanics were not significantly different from whites, except in the multivariate logistic regression analysis of the subset with CD4 counts $(\mathrm{OR}=0 \cdot 8,95 \%$ CI $0 \cdot 6,1 \cdot 0)$. One possible explanation for observing a lower risk of cryptosporidiosis in African Americans could be that they are more likely than whites to be IDUs than homosexual/bisexual $[13,14]$. Since cryptosporidiosis is less prevalent among IDUs than among MSM $[9,10,15]$, confounding by sexual orientation, as a surrogate for sexual transmission (i.e. the transmission of Cryptosporidium during sexual acts that involve faecal-oral contact), might account for the observed lower risk in this group. However, our analyses confirm that there remains a significantly lower risk for African Americans even after taking into account IDU and other covariates.

Other possible explanations for this finding could be related to host genetic factors, cultural and dietary norms, or other exposures to Cryptosporidium that could contribute to immunity at an earlier stage in life. A study in New York found that African Americans who were HIV positive were more likely to avoid drinking tap water than other racial/ethnic groups [16]. The observation that whites are at a higher risk than African Americans for cryptosporidiosis is a notable result because incidence rates of most infectious diseases are higher among African Americans than whites $[17,18]$.

African American AIDS patients are likely to have poorer access to care than whites [19-21], which might result in fewer cases of cryptosporidiosis, and AIDS in general, being diagnosed and treated for this group. However, this potential diagnostic bias should balance out by fewer African Americans diagnosed overall with AIDS. Studies have found that African Americans are less likely to be put on drug therapy for AIDS and related opportunistic infections [19-21]. Therefore, one would expect more cases of opportunistic infections rather than fewer should be found in this demographic group.

Surveillance data provide a means of exploring demographic characteristics that may be proxies for other associated risks. The trend of decreasing risk with increasing age in this study confirm the general finding that acquisition of cryptosporidiosis is agerelated possibly due to differential routes of exposure to the pathogen $[2,4,7]$. These data also corroborate earlier findings of an increased risk for cryptosporidiosis among homosexual/bisexual males [6,9]. One plausible explanation is that oral-anal contact is highly prevalent among this group (versus heterosexuals) which could be the route of sexual transmission of Cryptosporidium. However, from these data we are unable to determine which specific sexual practices may be responsible for the transmission of this parasite. Although studies have pointed specifically to anal-penile sexual contact [22], only a detailed prospective study would provide adequate insights. It is vital, particularly for those who are already infected with HIV, to realize that cryptosporidiosis is not only a waterborne infection. A recent inquiry into behavioural norms among HIVinfected patients in San Francisco found that although avoidance of tap water was high $(78 \%)$, high-risk sexual behaviour was common (41\%) [23].

One limitation of our study is that all the known risk factors for transmission of Cryptosporidium could not be assessed-specifically, water sources and related waterborne risks could not be determined from the available data. Thus residual confounding has not been entirely eliminated and may account for our observation of lower risk among African Americans if waterborne exposure is linked to race/ethnicity. Nevertheless, the fact that the logistic models fit our data fairly well indicates that the extent of this bias is probably not large enough to change the results significantly. Furthermore, a prior study in Los Angeles county found that municipal water supply was not a significant risk factor for cryptosporidiosis in AIDS patients [24].

Other inherent problems with these surveillance data include incompleteness of reporting and possible measurement error due to misclassification. In selecting only presenting cases of cryptosporidiosis for analysis we have not studied the full spectrum of the infection in AIDS patients; but by limiting our analysis to initial ADCs we have circumvented a reporting bias that may have been introduced due to extremely differential follow-up for subsequent conditions across local health jurisdictions. Race/ ethnicity is not self-reported and could represent a bias in our results if grossly misclassified. However, a 
study aimed at determining the extent of misclassification of race/ethnicity in the national AIDS surveillance data showed that race classification was in excellent agreement (over 96\%) with death certificate and with self-reports [25]. Another investigation into the reliability and validity of AIDS surveillance data found that both race and gender were in excellent agreement (kappa statistic 0.86 and $0 \cdot 97$, respectively) with medical records [26].

Cryptosporidiosis cannot be neglected as an important cause of HIV-related severe illness. Our study findings suggest that the white segment of the AIDS population in California is at a higher risk than African Americans for cryptosporidiosis even after accounting for other covariates. The possibility that there may be biological factors or differential lifetime exposures that account for the difference between the $\mathrm{racial} / \mathrm{ethnic}$ groups merits further investigation.

\section{ACKNOWLEDGEMENTS}

This work was supported by the California Department of Health Services Office of AIDS under contract number 00-911-80. This work has been submitted in partial fulfilment of the Ph.D. degree at the University of California, Berkeley.

\section{REFERENCES}

1. Current WL, Reese NC, Ernst JV, Bailey WS, Heyman MB, Weinstein WM. Human cryptosporidiosis in immunocompetent and immunodeficient persons. Studies of an outbreak and experimental transmission. N Engl J Med 1983; 308: 1252-7.

2. Guerrant RL. Cryptosporidiosis: an emerging, highly infectious threat. Emerg Infect Dis 1997; 3: 51-7.

3. Petersen C. Cryptosporidiosis in patients infected with the human immunodeficiency virus. Clin Infect Dis 1992; 15: 903-9.

4. Juranek DD. Cryptosporidiosis: sources of infection and guidelines for prevention. Clin Infect Dis 1995; 21 (Suppl 1): S57-61.

5. Manabe YC, Clark DP, Moore RD, et al. Cryptosporidiosis in patients with AIDS: correlates of disease and survival. Clin Infect Dis 1998; 27: 536-42.

6. Sorvillo FJ, Lieb LE, Kerndt PR, Ash LR. Epidemiology of cryptosporidiosis among persons with acquired immunodeficiency syndrome in Los Angeles County. Am J Trop Med Hyg 1994; 51 : 326-31.

7. Sorvillo F, Beall G, Turner PA, et al. Seasonality and factors associated with cryptosporidiosis among individuals with HIV infection. Epidemiol Infect 1998; 121 : 197-204.

8. Colford JM, Jr., Tager IB, Hirozawa AM, Lemp GF, Aragon T, Petersen C. Cryptosporidiosis among patients infected with human immunodeficiency virus.
Factors related to symptomatic infection and survival. Am J Epidemiol 1996; 144: 807-16.

9. Pedersen C, Danner S, Lazzarin A, et al. Epidemiology of cryptosporidiosis among European AIDS patients. Genitourin Med 1996; 72: 128-31.

10. Jones JL, Hanson DL, Dworkin MS, et al. Surveillance for AIDS-defining opportunistic illnesses, 1992-1997. MMWR CDC Surveillance Summaries 1999; 48: 1-22.

11. Hosmer DW, Lemeshow S. Applied logistic regression. New York, NY: John Wiley and Sons, 1989.

12. Hashmey R, Smith NH, Cron S, Graviss EA, Chappell CL, White AC, Jr. Cryptosporidiosis in Houston, Texas. A report of 95 cases. Medicine (Baltimore) 1997; 76: 118-39.

13. Jones JL, Hanson DL, Dworkin MS, Kaplan JE, Ward JW. Trends in AIDS-related opportunistic infections among men who have sex with men and among injecting drug users 1991-1996. J Infect Dis 1998; 178: 114-20.

14. Facer M, Jungkeit M, Molitor F. AIDS among racial/ethnic groups in California, 1998 ed. Sacramento, CA: California Department of Health Services, Office of AIDS, Epidemiologic Studies Section, 1999.

15. Lopez-Velez R, Tarazona R, Garcia Camacho A, et al. Intestinal and extraintestinal cryptosporidiosis in AIDS patients. Eur J Clin Microbiol Infect Dis 1995; 14: 677-81.

16. Davis LJ, Roberts HL, Juranek DD, Framm SR, Soave R. A survey of risk factors for cryptosporidiosis in New York City: drinking water and other exposures. Epidemiol Infect 1998; 121: 357-67.

17. Cooper RS. Health and the social status of blacks in the United States. Ann Epidemiol 1993; 3: 137-44.

18. Ernster VL, Colford JMJ. Host and disease: influence of demographic and socioeconomic factors. In: Fauci AS, ed. Harrison's principles of internal medicine, 14th ed. New York: McGraw-Hill, 1998: 96-133.

19. Sackoff J, McFarland J, Su S, Bryan E. Prophylaxis for opportunistic infections among HIV-infected patients receiving medical care. J Acquir Immune Defic Syndr Hum Retrovirol 1998; 19: 387-92.

20. Moore RD, Stanton D, Gopalan R, Chaisson RE. Racial differences in the use of drug therapy for HIV disease in an urban community. N Engl J Med 1994; 330: 763-8.

21. Easterbrook PJ, Keruly JC, Creagh-Kirk T, Richman DD, Chaisson RE, Moore RD. Racial and ethnic differences in outcome in zidovudine-treated patients with advanced HIV disease. Zidovudine Epidemiology Study Group. JAMA 1991; 266: 2713-8.

22. Esfandiari A, Jordan WC, Brown CP. Prevalence of enteric parasitic infection among HIV-infected attendees of an inner city AIDS clinic. Cell Mol Biol (Noisy-le-grand) 1995; 41 (Suppl 1): S19-23.

23. Kim LS, Stansell J, Cello JP, Koch J. Discrepancy between sex- and water-associated risk behaviors for cryptosporidiosis among HIV-infected patients in San Francisco. J Acquir Immune Defic Syndr Hum Retrovirol 1998; 19: 44-9.

24. Sorvillo F, Lieb LE, Nahlen B, Miller J, Mascola L, 
Ash LR. Municipal drinking water and cryptosporidiosis among persons with AIDS in Los Angeles County. Epidemiol Infect 1994; 113: 313-20.

25. Kelly JJ, Chu SY, Diaz T, Leary LS, Buehler JW. Race/ethnicity misclassification of persons reported with AIDS. The AIDS Mortality Project Group and
The Supplement to HIV/AIDS Surveillance Project Group. Ethn Health 1996; 1: 87-94.

26. Gallagher KM, Demaria A, Jara M. Case report vs. medical record: the validity and reliability of AIDS surveillance data. 4th Conference on Retroviral and Opportunistic Infections, 1997: abstract 356. 